\title{
Review Article \\ HBT Radii: Comparative Studies on Collision Systems and Beam Energies
}

\author{
Debasish Das \\ Saha Institute of Nuclear Physics, HBNI, 1/AF, Bidhannagar, Kolkata 700064, India \\ Correspondence should be addressed to Debasish Das; dev.deba@gmail.com
}

Received 12 February 2018; Revised 14 June 2018; Accepted 28 June 2018; Published 15 July 2018

Academic Editor: Fu-Hu Liu

Copyright (C) 2018 Debasish Das. This is an open access article distributed under the Creative Commons Attribution License, which permits unrestricted use, distribution, and reproduction in any medium, provided the original work is properly cited. The publication of this article was funded by SCOAP $^{3}$.

\begin{abstract}
Two-particle Hanbury-Brown-Twiss (HBT) interferometry is an important probe for understanding the space-time structure of particle emission sources in high energy heavy ion collisions. We present the comparative studies of $\mathrm{HBT}$ radii in $\mathrm{Pb}+\mathrm{Pb}$ collisions at $\sqrt{s_{\mathrm{NN}}}=17.3 \mathrm{GeV}$ with $\mathrm{Au}+\mathrm{Au}$ collisions at $\sqrt{s_{\mathrm{NN}}}=19.6 \mathrm{GeV}$. To further understand this specific energy regime, we also compare the $\mathrm{HBT}$ radii for $\mathrm{Au}+\mathrm{Au}$ collisions at $\sqrt{s_{\mathrm{NN}}}=19.6 \mathrm{GeV}$ with $\mathrm{Cu}+\mathrm{Cu}$ collisions at $\sqrt{s_{\mathrm{NN}}}=22.4 \mathrm{GeV}$. We have found interesting similarity in the $R_{\text {out }} / R_{\text {side }}$ ratio with $m_{\mathrm{T}}$ across the collision systems while comparing the data for this specific energy zone which is interesting as it acts as a bridge from SPS energy regime to the RHIC energy domain.
\end{abstract}

\section{Introduction}

A phase transition from a hadronic state to a "plasma" of deconfined quarks and gluons when the energy density exceeds a critical value is predicted from Quantum ChromoDynamics (QCD). The complicated structure of nuclear matter at low temperatures, where it is composed of a multitude of hadronic particles, baryons, and mesons, is thus expected to give way at high temperatures to a plasma of weakly composed quarks and gluons, the Quark-Gluon Plasma (QGP). QGP is a thermalized system where the properties of the system are governed by the quark and gluon degrees of freedom [1].

Understanding the deconfining phase transition in hadronic matter and the QGP properties is a challenging task. For systems created in the relativistic heavy ion collider (RHIC) and large hadron collider (LHC), energy region with high temperatures, and low baryon-chemical potential, Lattice QCD calculations predict a crossover transition between the hadron gas and the QGP phase. Lattice QCD predicts a phase transformation to a quark-gluon plasma at a temperature of approximately $T \approx 170 \mathrm{MeV}(1 \mathrm{MeV} \approx$ $\left.1.1604 \times 10^{10} \mathrm{~K}\right)([1])$ corresponding to an energy density $\epsilon \approx 1 \mathrm{GeV} / \mathrm{fm}^{3}$, which is nearly an order of magnitude larger than normal nuclear matter.
Experimental studies in relativistic heavy ion physics aim to study the QCD nature of matter under the conditions of extreme temperature and high energy density both at RHIC and at LHC. The discovery of the QGP can describe the system (governed by the quarks and gluons) in which the degrees of freedom are no more the color neutral hadron states.

The equation of state (EoS) of nuclear matter enables us to understand the relationship between the pressure and the energy at a given net-baryon density. Phase transitions from the hadronic resonance gas phase to the color-deconfined QGP (see, e.g., $[2,3]$ ) contribute to the changes of the EoS. The experimental measurements should also be able to determine the physical characteristics of the transition, for example, the critical temperature, the order of the phase transition, and the speed of the sound along with the nature of the quasi-particles. The EoS of hot and dense QCD matter is still not precisely understood. Modern nuclear physics, has an important goal, that is, to explore the phase diagram of quark matter in various temperatures and baryon density so as to confirm the existence of the new phase of quark matter $[4,5]$.

The intermediate Super Proton Synchrotron (SPS) energy regime still remains interesting since the onset of deconfinement is expected to happen at those energies. Possibility of a 
critical endpoint $[6,7]$ and a first-order phase transition is yet not excluded. Several beam-energy dependent observables such as the particle ratios $[8,9]$, the flow $[10,11]$, and the HBT parameters $[12,13]$ show a nonmonotonic behavior, for which the interpretation still remains unclear. The beamenergy scan (BES) programs at RHIC show that directed flow is strong for both the lowest and the highest RHIC energies as shown by results from STAR experiment [14]. The netproton $v_{1}(y)$ slope has a minimum between 11.5 and $19.6 \mathrm{GeV}$ and changing sign twice between 7.7 and $39 \mathrm{GeV}$, which is quite contrary to the UrQMD transport model predictions for that energy regime. The vanishing of directed flow when the expansion stops and its appearance when the matter has passed through the change constitute the "latent heat", where the predicted "softest point disappearance" of flow can become a possible signature of a first-order phase transition between hadronic matter and a deconfined QGP phase.

Assuming a first-order phase transition, there is a mixed phase of the QGP and hadronic gas. A slow-burning fireball is expected in the absence of pressure gradient, when the initial system is at rest in the mixed phase, and this leads to a timedelay in the system evolution [12, 15-17]. Investigation of the time-delay signatures for the first-order phase transition is henceforth a subject of interest.

Two-particle Hanbury-Brown-Twiss (HBT) interferometry is an important tool for detecting the space-time structure of particle emission sources in high energy heavy ion collisions [18-20]. The occurrence of first-order phase transition between the QGP and hadronic matter will lead to the time-delay of the system evolution, hence making the emission duration of particles more prolonged [12, 15-17]. As explained in $[12,15-17]$ the three HBT radius parameters, $R_{\text {out }}, R_{\text {side }}, R_{\text {long }}$, describe the dimensions of a Gaussian source in longitudinal comoving system (LCMS) framework. The $R_{\text {out }} / R_{\text {side }}$ ratio can be related to the emission time $[12,15-$ 17]. We have explored in this paper the energy region of 17.3 $\mathrm{GeV}$ to $22.4 \mathrm{GeV}$ through comparative studies of two-pion HBT radii. This energy region has shown interesting results in STAR experiment [14] for other correlation measurements (like flow).

\section{Results}

The intensity interferometry technique for measuring sizes of stars [21] was formulated by Robert Hanbury Brown and Richard Twiss and is also known as the "Hanbury-BrownTwiss (HBT) effect". Such technique was extended to particle physics [22] for understanding the angular distributions of pion pairs in $p \bar{p}$ annihilations and thus the quantum statistics causing an enhancement in pairs with low relative momentum. In HBT analyses the method has henceforth evolved into a precision tool for measuring the space-time properties of the regions of homogeneity at kinetic freeze-out in heavy ion collisions [23].

Two-pion interferometry yields HBT radii that describe the geometry of these regions of homogeneity (regions that emit correlated pion pairs). The HBT radii increase for more central collisions due to the increasing volume of the source and hence demonstrate how HBT can probe spatial sizes and shapes [24]. The decrease of HBT radii with mean pair transverse momentum, $\left.k_{T}\left(=\left|\vec{p}_{1 \mathrm{~T}}+\vec{p}_{2 \mathrm{~T}}\right|\right) / 2\right)$, has been due to transverse and longitudinal flow [24]. Flow causes space-momentum correlations since the sizes of the regions emitting the particles do not correspond to the entire fireball created in a relativistic heavy ion collision [24].

In this paper, the results of two-pion HBT analyses of $\mathrm{Pb}+\mathrm{Pb}$ at $17.3 \mathrm{GeV}$ from NA49 experiment [25] are compared in Figure 1 and discussed with other STAR HBT results from $\mathrm{Au}+\mathrm{Au} 19.6 \mathrm{GeV}$ [26]. Figure 1 shows the HBT radii of SPS and RHIC collision species where $\mathrm{Pb}+\mathrm{Pb} 17.3 \mathrm{GeV}$ (NA49) and $\mathrm{Au}+\mathrm{Au} 19.6 \mathrm{GeV}(\mathrm{STAR})$ show similar trend for $R_{\text {side }}$ and $R_{\text {long }}$ with $m_{\mathrm{T}}$. For $R_{\text {out }}$ the SPS data has a flatter slope when compared with RHIC, but the $R_{\text {out }} / R_{\text {side }}$ ratios with $m_{\mathrm{T}}$ $\left(=\sqrt{k_{\mathrm{T}}^{2}+m_{\pi}^{2}}\right)$ are very similar for the top central data of both experiments. The $R_{\text {out }} / R_{\text {side }}$ ratios of NA49 and STAR show weak $m_{\mathrm{T}}$ dependence and have values close to unity.

The HBT radii from $\mathrm{Au}+\mathrm{Au} 19.6 \mathrm{GeV}$ and $\mathrm{Cu}+\mathrm{Cu} 22.4$ $\mathrm{GeV}$, both from STAR experiment, are also included in this paper since they are different collision species with close collision energies. Reference [27] explains the analysis methodology for $\mathrm{Cu}+\mathrm{Cu}$ collisions at $\sqrt{s_{\mathrm{NN}}}=22.4 \mathrm{GeV}$. In Figure 2 we present this comparison of two-pion $\mathrm{HBT}$ radii to include central (0-5\%) Au+Au collisions at $\sqrt{s_{\mathrm{NN}}}=19.6 \mathrm{GeV}$ and central $(0-10 \%) \mathrm{Cu}+\mathrm{Cu}$ collisions at $\sqrt{s_{\mathrm{NN}}}=22.4 \mathrm{GeV}$ from the STAR experiment.

The $\mathrm{HBT}$ radii for $\mathrm{Cu}+\mathrm{Cu}$ collisions at $\sqrt{s_{\mathrm{NN}}}=22.4 \mathrm{GeV}$ are smaller than those for $\mathrm{Au}+\mathrm{Au}$ collisions at $\sqrt{s_{\mathrm{NN}}}=19.6$ $\mathrm{GeV}$. The variations of the $R_{\text {out }} / R_{\text {side }}$ ratios with $m_{\mathrm{T}}$ are similar for the $\mathrm{Au}+\mathrm{Au}$ and $\mathrm{Cu}+\mathrm{Cu}$ collision data as we see in Figure 2. The ratios also show weak $m_{\mathrm{T}}$ dependence with the values close to unity.

In Figure 3 we present the $m_{\mathrm{T}}$ dependence of the ratios of two-pion $\mathrm{HBT}$ radii for the most-central $\mathrm{Au}+\mathrm{Au}$ at $\sqrt{s_{\mathrm{NN}}}=19.6 \mathrm{GeV}$ and $\mathrm{Cu}+\mathrm{Cu}$ collisions at $\sqrt{s_{\mathrm{NN}}}=22.4 \mathrm{GeV}$. Details about the $\mathrm{Cu}+\mathrm{Cu}$ systems are explained in [27] and references therein. As seen in Figure 3 the ratios of radii for $\mathrm{Au}+\mathrm{Au}$ to those for $\mathrm{Cu}+\mathrm{Cu}$ collisions are $\sim 1.5$. Although we see that the individual HBT radii decrease significantly with increasing $m_{\mathrm{T}}$, the ratios in Figure 3 show that the HBT radii for $\mathrm{Au}+\mathrm{Au}$ and $\mathrm{Cu}+\mathrm{Cu}$ collisions at $19.6 \mathrm{GeV}$ and 22.4 $\mathrm{GeV}$ share a common $m_{\mathrm{T}}$ dependence. Such trends can be understood in terms of models $[28,29]$ where participant scaling is used to predict the $\mathrm{HBT}$ radii in $\mathrm{Cu}+\mathrm{Cu}$ collisions from the measured radii for $\mathrm{Au}+\mathrm{Au}$ collisions at $\sqrt{s_{\mathrm{NN}}}=200$ $\mathrm{GeV}$, assuming the radii are proportional to $\mathrm{A}^{1 / 3}$, where $\mathrm{A}$ is the atomic mass number of the colliding nuclei.

\section{Summary}

The $R_{\text {out }} / R_{\text {side }}$ ratio is important since it is able to provide the information of the emission duration. We also know that the HBT radii are affected by transverse and longitudinal flow. The SPS energy regime is still zone of interest where the recent flow results from STAR experiment [14] (within 11.5 and $19.6 \mathrm{GeV}$ ) have shown some new and interesting features. When we compare the HBT (two-particle correlation) radii in $\mathrm{Pb}+\mathrm{Pb}$ collisions at $\sqrt{s_{\mathrm{NN}}}=17.3 \mathrm{GeV}$ with $\mathrm{Au}+\mathrm{Au}$ collisions at 
19.6 GeV Au+Au and 17.3 GeV Pb+Pb

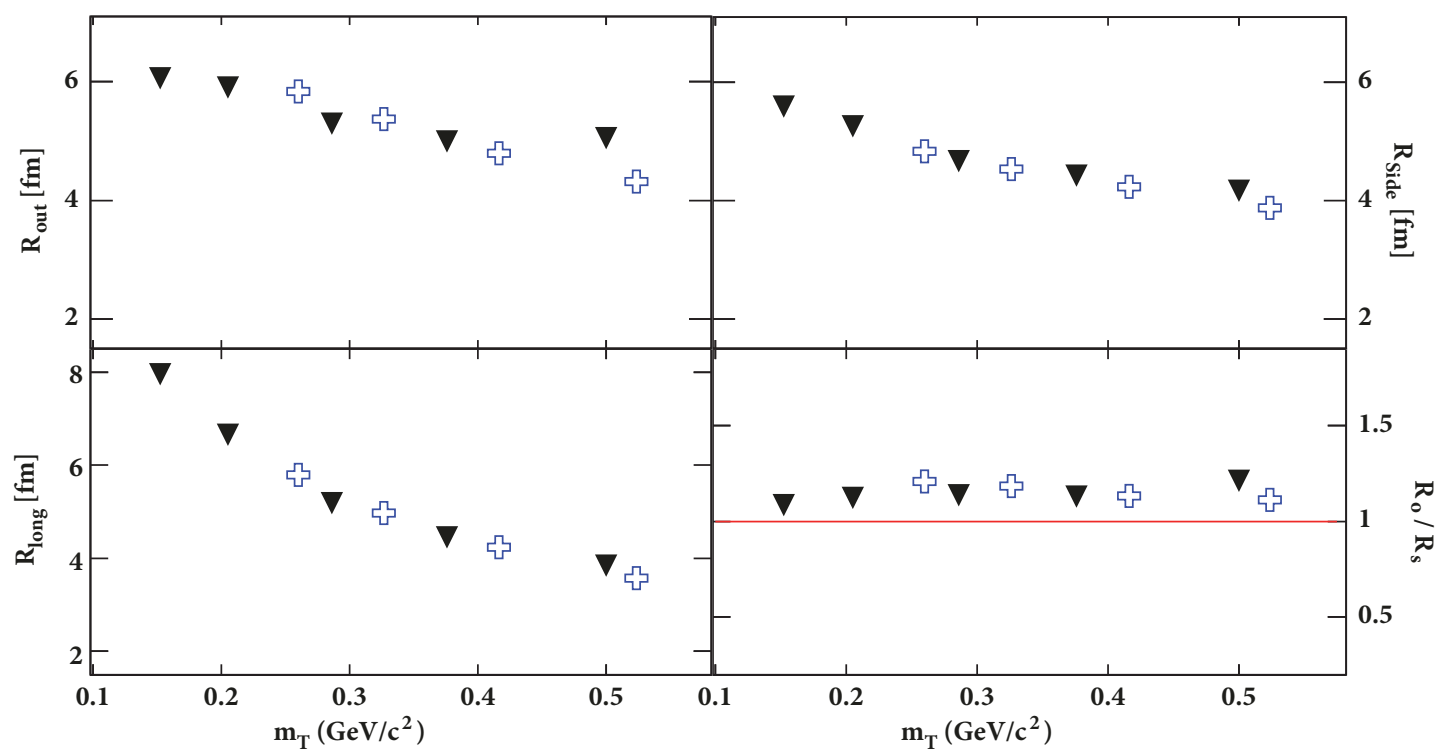

§ 19.6 GeV Au+Au (0-5\%) [STAR Published in Phys. Rev. C 92, 014904 (2015)]

$\nabla$ 17.3 GeV Pb+Pb (most central) [NA49 Published in Phys. Rev. C77 (2008) 064908]

Figure 1: The comparison of system size dependence in HBT radii of STAR Au+Au collisions at $\sqrt{s_{\mathrm{NN}}}=19.6 \mathrm{GeV}$ with NA49 $\mathrm{Pb}+\mathrm{Pb}$ collisions for $17.3 \mathrm{GeV}$. Only statistical errors are shown for the top central data of both experiments.

\subsection{GeV Au+Au and 22.4 GeV Cu+Cu}

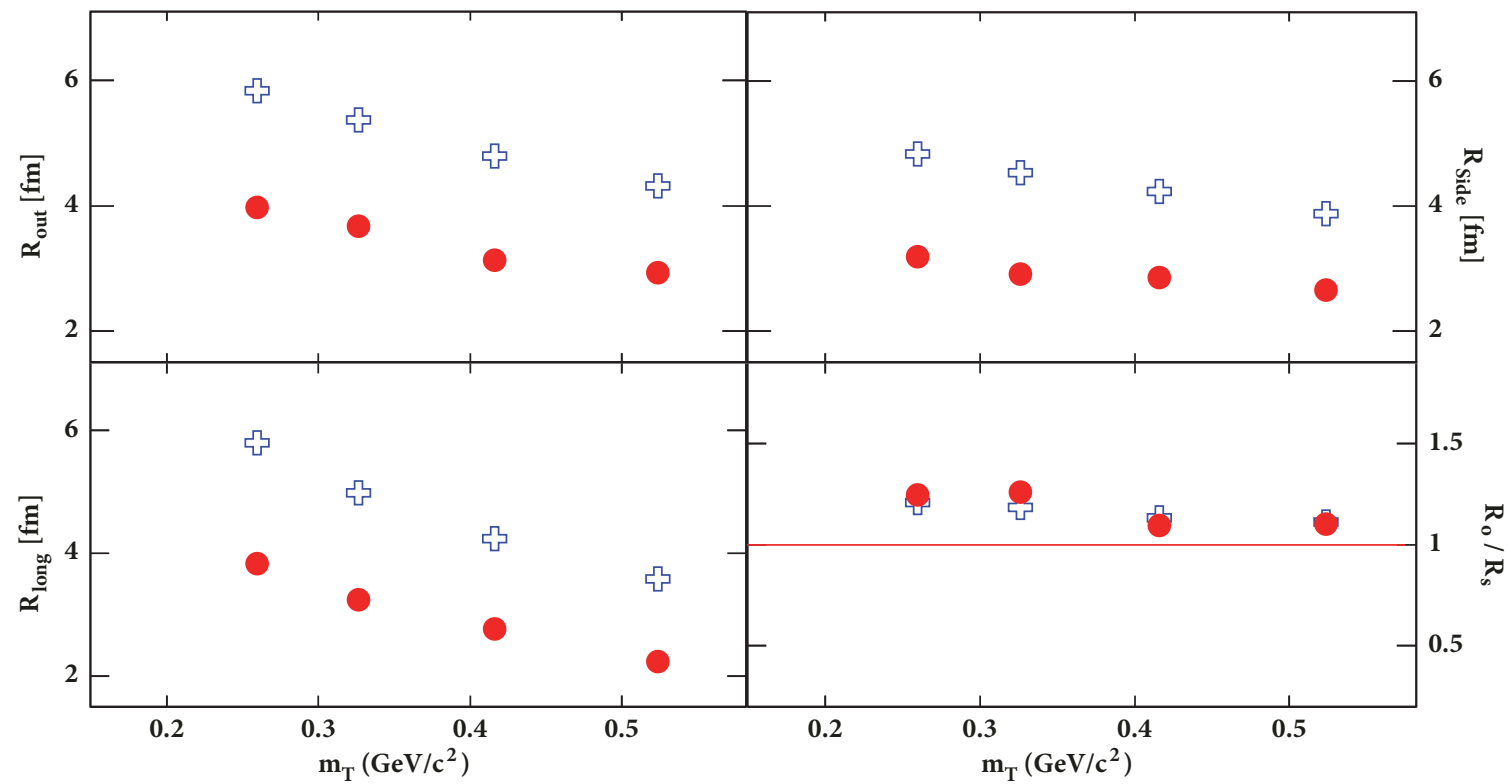

५ $19.6 \mathrm{GeV}$ Au+Au (0-5\%) [STAR Published in Phys. Rev. C 92, 014904 (2015)]

22.4 GeV Cu+Cu (0-10\%) [STAR Preliminary in DAE Symp. Nucl. Phys. 54 (2009) 540-541]

Figure 2: The comparison of system size dependence in HBT radii of STAR Au+Au collisions at $\sqrt{s_{\mathrm{NN}}}=19.6 \mathrm{GeV}$ with $\mathrm{Cu}+\mathrm{Cu}$ collisions at $\sqrt{s_{\mathrm{NN}}}=22.4 \mathrm{GeV}$. Only statistical errors are shown for the top central data of both the $\mathrm{Au}+\mathrm{Au}$ and $\mathrm{Cu}+\mathrm{Cu}$ datasets. 

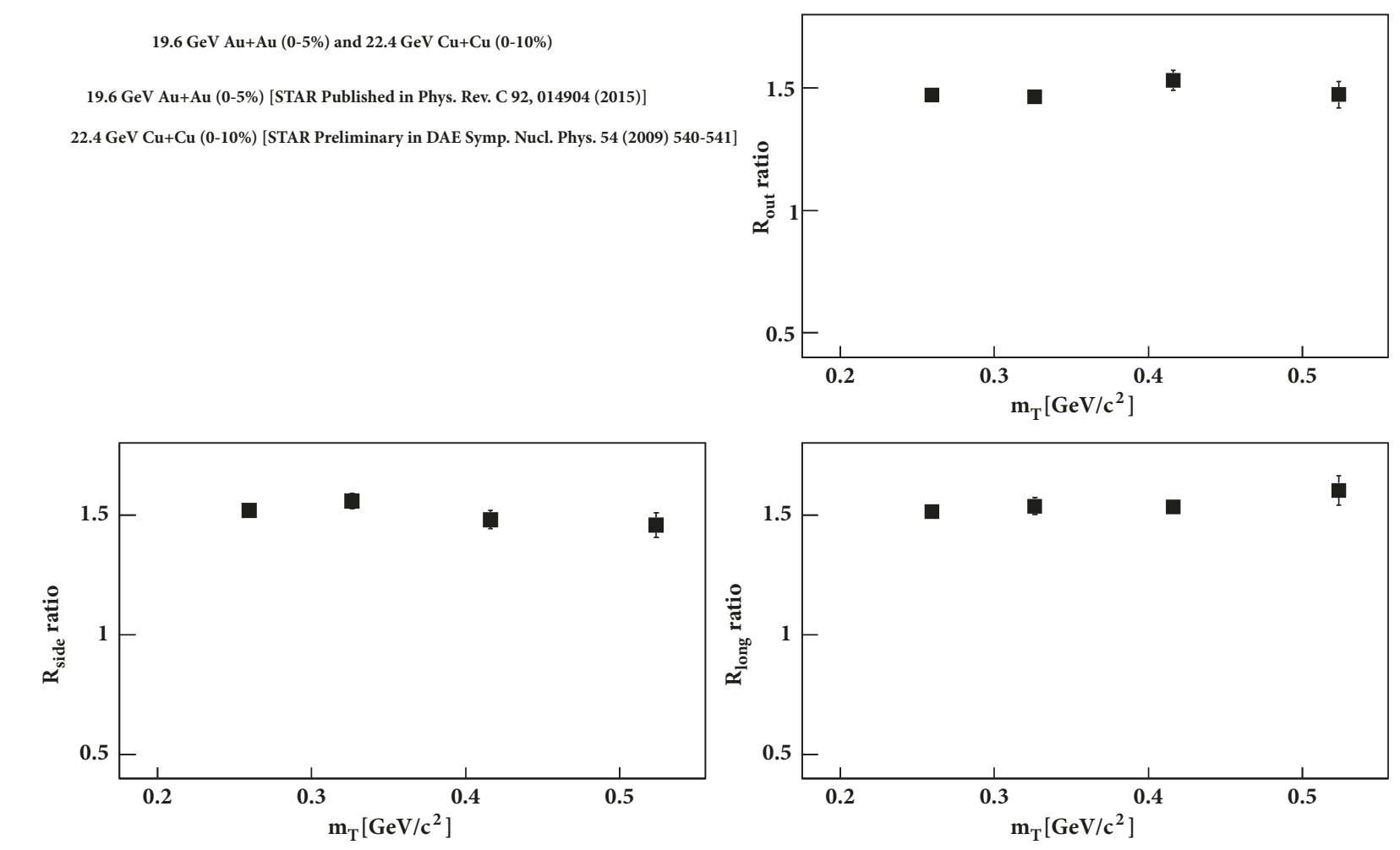

$19.6 \mathrm{GeV} \mathrm{Au}+\mathrm{Au}[0-5 \%] / 22.4 \mathrm{GeV} \mathrm{Cu}+\mathrm{Cu}[0-10 \%]$

FIgURE 3: Ratios of HBT radii at top centralities for $\mathrm{Au}+\mathrm{Au}$ and $\mathrm{Cu}+\mathrm{Cu}$ collisions at $\sqrt{s_{\mathrm{NN}}}=19.6$ and $22.4 \mathrm{GeV}$ versus $m_{T}$. Only statistical errors are shown for $\mathrm{Au}+\mathrm{Au}$ collisions at $\sqrt{s_{\mathrm{NN}}}=19.6 \mathrm{GeV}$ and $\mathrm{Cu}+\mathrm{Cu}$ collisions at $\sqrt{s_{\mathrm{NN}}}=22.4$ for their top central datasets.

$\sqrt{s_{\mathrm{NN}}}=19.6 \mathrm{GeV}$, we find very similar $R_{\text {out }} / R_{\text {side }}$ ratio with $m_{\mathrm{T}}$. To explore this interesting energy regime we have compared the HBT radii for $\mathrm{Au}+\mathrm{Au}$ collisions at $\sqrt{s_{\mathrm{NN}}}=19.6 \mathrm{GeV}$ with $\mathrm{Cu}+\mathrm{Cu}$ collisions at $\sqrt{s_{\mathrm{NN}}}=22.4 \mathrm{GeV}$. The similarity in the $R_{\text {out }} / R_{\text {side }}$ ratio with $m_{\mathrm{T}}$ persists across the collision systems from SPS to RHIC energies and even in close RHIC energies for $\mathrm{Au}+\mathrm{Au}$ and $\mathrm{Cu}+\mathrm{Cu}$ systems as well. The rise of the ratio $R_{\text {out }} / R_{\text {side }}$ with collision energy which was predicted [12] due to a possible phase transition is not observed. Such inferences establish that HBT radii $R_{\text {out }} / R_{\text {side }}$ ratios are very much comparable and consistent across the different colliding species in (an exciting zone of interest of the RHIC BES program) the energy region of $17.3 \mathrm{GeV}$ to $22.4 \mathrm{GeV}$.

\section{Conflicts of Interest}

The author declares that there are no conflicts of interest regarding the publication of this paper.

\section{Acknowledgments}

The author acknowledges the facilities of Saha Institute of Nuclear Physics, Kolkata, India.

\section{References}

[1] F. Karsch, "Lecture Notes in Physics," in Nuclear Physics A, F. Karsch, Ed., vol. 698, p. 199, 2002.
[2] C. Spieles, H. Stöcker, and C. Greiner, "Phase transition of a finite quark-gluon plasma," Physical Review C: Nuclear Physics, vol. 57, no. 2, pp. 908-915, 1998.

[3] M. Bluhm, B. Kämpfer, R. Schulze, D. Seipt, and U. Heinz, "A Family of equations of state based on lattice QCD: impact on flow in ultrarelativistic heavy-ion collisions," Physical Review C: Nuclear Physics, vol. 76, no. 3, Article ID 034901, 2007.

[4] Z. Fodor and S. D. Katz, "Lattice determination of the critical point of QCD at finite T and $\mu$," Journal of High Energy Physics, vol. 2002, no. 3, p. 14, 2002.

[5] P. d. Forcrand and O. Philipsen, "The chiral critical line of," Journal of High Energy Physics, vol. 2007, no. 1, pp. 77-77, 2007.

[6] R. A. Lacey, N. N. Ajitanand, J. M. Alexander et al., "Has the QCD Critical Point Been Signaled by Observations at the BNL Relativistic Heavy Ion Collider?" Physical Review Letters, vol. 98, Article ID 092301, 2007.

[7] "Searching for Minimum in Dependence of Squared Speed-ofSound on Collision Energy," Advances in High Energy Physics, vol. 2016, Article ID 9467194, 9 pages, 2016.

[8] S. V. Afanasiev, "Energy dependence of pion and kaon production in central $\mathrm{Pb}+\mathrm{Pb}$ collisions," Physical Review C, vol. 66, Article ID 054902, 2002.

[9] C. Alt et al., "Pion and kaon production in central $\mathrm{Pb}+\mathrm{Pb}$ collisions at $20 \mathrm{~A}$ and $30 \mathrm{~A} \mathrm{GeV}$ : Evidence for the onset of deconfinement," Physical Review C, vol. 77, no. 4, Article ID 024903, 2008.

[10] P. F. Kolb, P. Huovinen, U. Heinz, and H. Heiselberg, "Elliptic flow at SPS and RHIC: from kinetic transport to hydrodynamics," Physics Letters B, vol. 500, no. 3-4, pp. 232-240, 2001. 
[11] H. Petersen, Q. Li, X. Zhu, and M. Bleicher, " Directed and elliptic flow in heavy-ion collisions from " Physical Review C: Nuclear Physics, vol. 74, no. 6, 2006.

[12] D. H. Rischke and M. Gyulassy, "The time-delay signature of quark-gluon plasma formation in relativistic nuclear collisions," Nuclear Physics A, vol. 608, pp. 479-512, 1996.

[13] D. Adamova et al., "Universal Pion Freeze-Out in Heavy-Ion Collisions," Physical Review Letters, vol. 90, Article ID 022301, 2003.

[14] L. Adamczyk, “Publisher's Note: Dielectron Mass Spectra from ," Physical Review Letters, vol. 113, no. 4, 2014.

[15] S. Pratt, "Pion interferometry of quark-gluon plasma," Physical Review D: Particles, Fields, Gravitation and Cosmology, vol. 33, Article ID 1314, 1986.

[16] G. Bertsch and G. E. Brown, "Temporal development of the plasma phase transition," Physical Review C: Nuclear Physics, vol. 40, no. 4, pp. 1830-1832, 1989.

[17] S. Soff, S. A. Bass, D. H. Hardtke, and S. Y. Panitkin, "Kaon Interferometry: A Sensitive Probe of the QCD Equation of State?" Physical Review Letters, vol. 88, no. 7, 2002.

[18] C. Wong, Introduction to High-Energy Heavy-Ion Collisions, World Scientific, 1994.

[19] U. A. Wiedemann and U. Heinz, "Particle interferometry for relativistic heavy-ion collisions," Physics Reports, vol. 319, no. 45, pp. 145-230, 1999.

[20] R. M. Weiner, "Boson interferometry in high-energy physics," Physics Reports, vol. 327, no. 5, pp. 249-346, 2000.

[21] R. Hanbury Brown and R. Q. Twiss, "A test of a new type of stellar interferometer on Sirius," Nature, vol. 178, no. 4541, pp. 1046-1048, 1956.

[22] G. Goldhaber, S. Goldhaber, W. Lee, and A. Pais, "Influence of Bose-Einstein statistics on the antiproton-proton annihilation process," Physical Review A: Atomic, Molecular and Optical Physics, vol. 120, no. 1, pp. 300-312, 1960.

[23] M. A. Lisa, S. Pratt, R. Soltz, and U. Wiedemann, "Femtoscopy in relativistic heavy ion collisions: two decades of progress," Annual Review of Nuclear and Particle Science, vol. 55, pp. 357402, 2005.

[24] S. Pratt and J. Vredevoogd, "Femtoscopy in relativistic heavy ion collisions and its relation to bulk properties of QCD matter," Physical Review C, vol. 78, Article ID 069901, 2008.

[25] C. Alt, "Bose-Einstein correlations of $\pi-\pi-$ pairs in central $\mathrm{Pb}+\mathrm{Pb}$ collisions at 20A,30A,40A,80A, and 158A GeV," Physical Review C, vol. 77, Article ID 064908, 2008.

[26] L. Adamczyk et al., "Beam-energy-dependent two-pion interferometry and the freeze-out eccentricity of pions measured in heavy ion collisions at the STAR detector," Physical Review C, vol. 92, 2015.

[27] D. Das, "Nuclear Physics," in Proceedings of the DAE International Symposium on Nuclear Physics, vol. 54, Bhabha Atomic Research Centre, Mumbai, India, 2009.

[28] J. G. Cramer, G. A. Miller, J. M. S. Wu, and J. H. S. Yoon, "Quantum Opacity, the RHIC Hanbury Brown-Twiss Puzzle, and the Chiral Phase Transition," Physical Review Letters, vol. 94, Article ID 102302, 2005.

[29] G. A. Miller and J. G. Cramer, "Polishing the lens: I. Pionic final state interactions and HBT correlations: distorted wave emission-function (DWEF) formalism and examples," Journal of Physics G: Nuclear and Particle Physics, vol. 34, p. 703, 2007. 

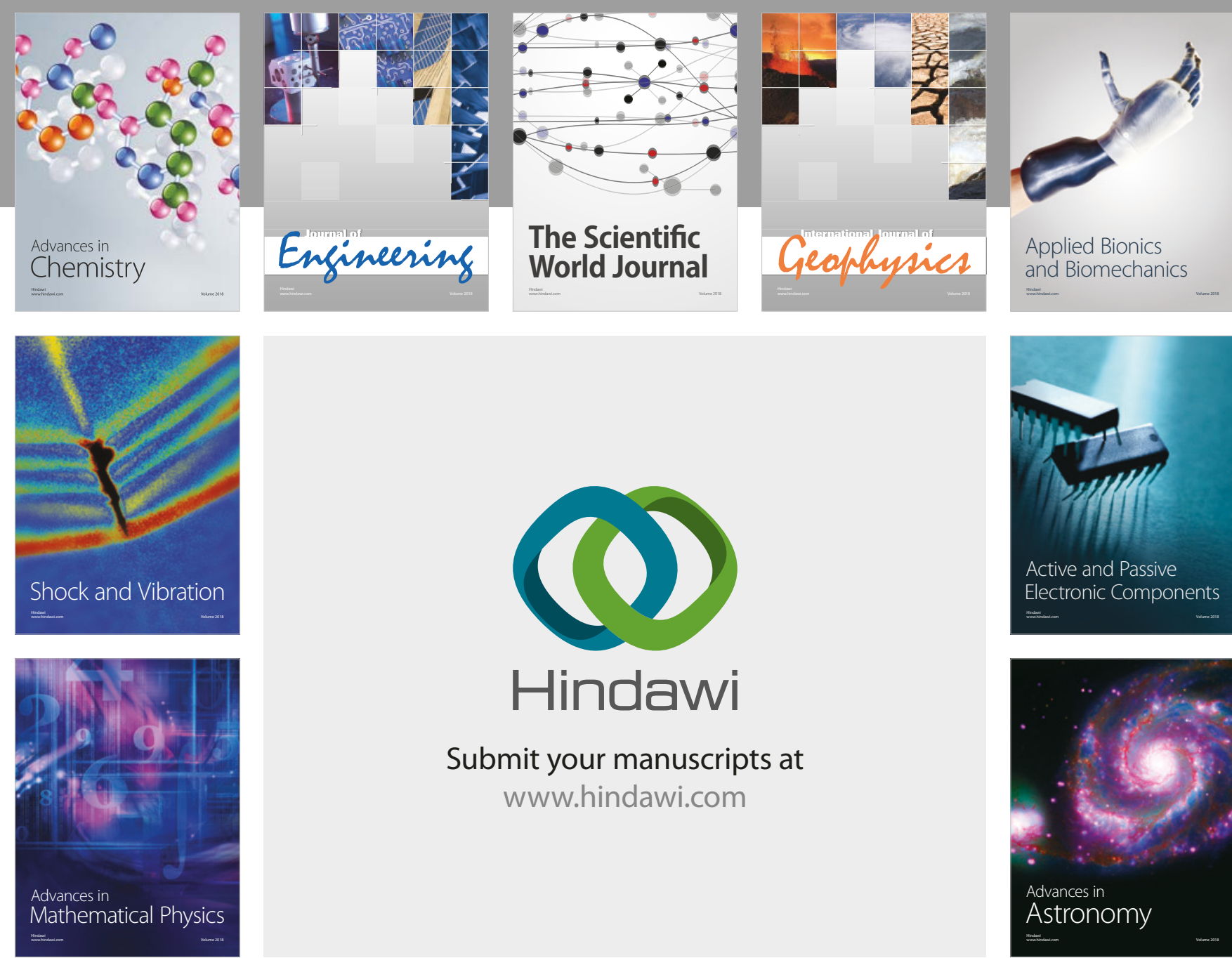

Submit your manuscripts at

www.hindawi.com

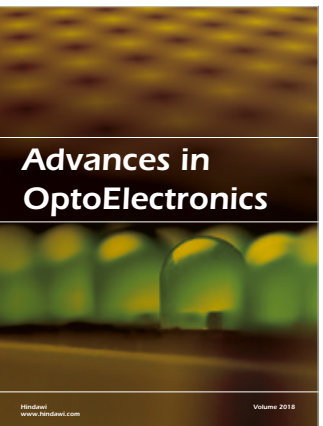

\section{Rotcting Machinery}
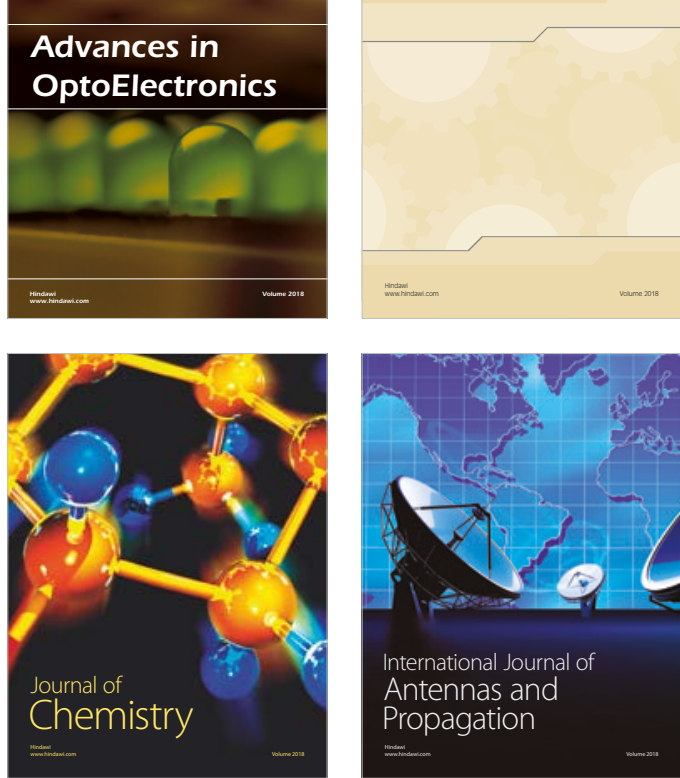

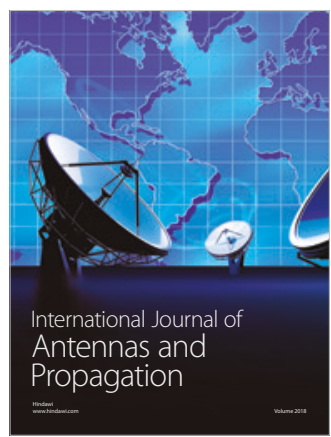

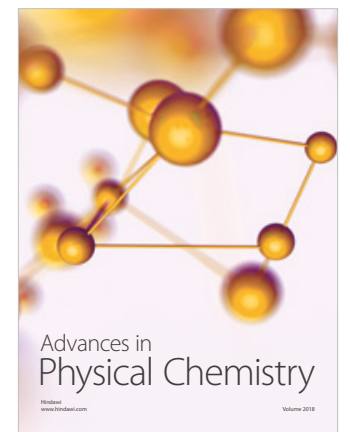

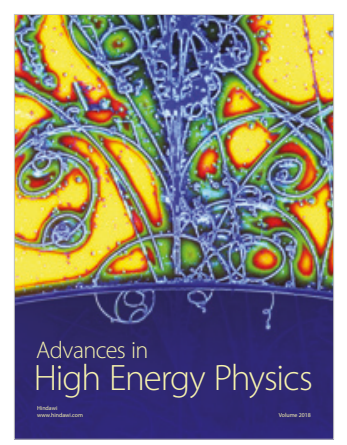

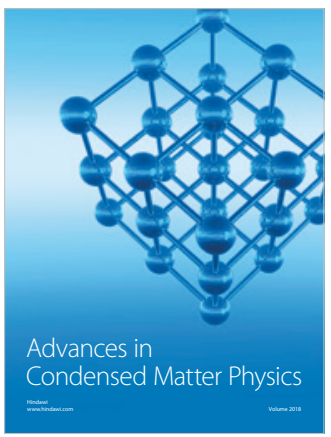

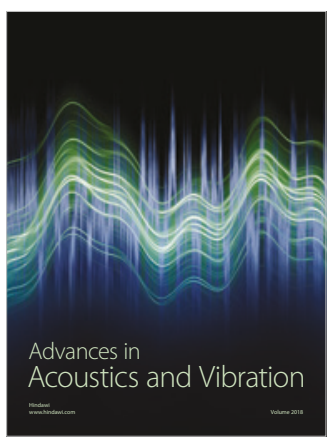

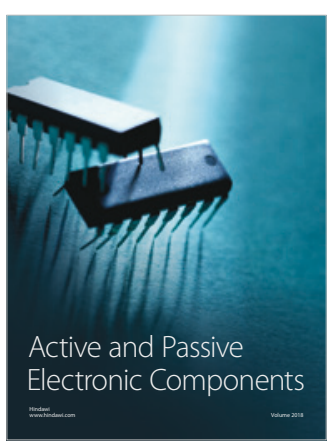
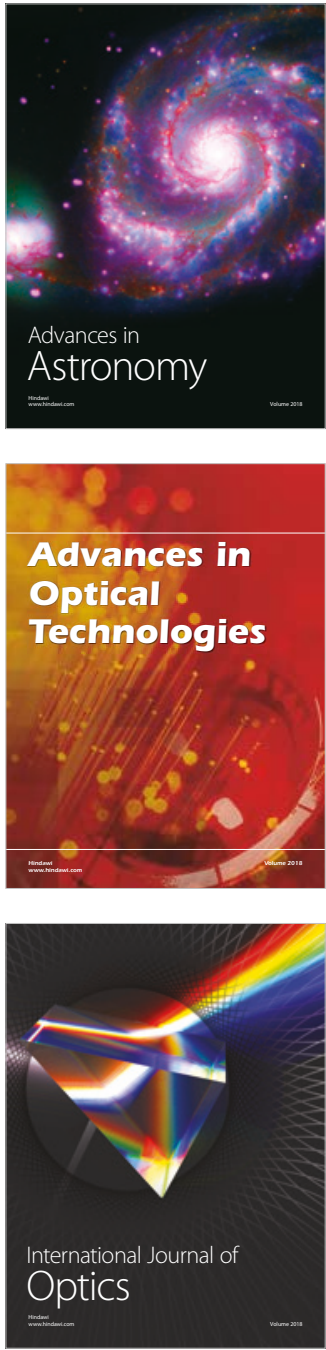